\title{
Uso de tecnologías inmersivas y su impacto en las actitudes científico-matemáticas del estudiantado de Educación Secundaria Obligatoria en un contexto en riesgo de exclusión social*
}

\author{
Francisco Silva-Díaz \\ Universidad de Granada. España. \\ fsilva@correo.ugr.es \\ Javier Carrillo-Rosúa \\ Universidad de Granada. España. \\ Instituto Andaluz de Ciencias de la Tierra (CSIC-UGR). España. \\ fjcarril@ugr.es \\ José Antonio Fernández-Plaza \\ Universidad de Granada. España. \\ joseanfplaza@ugr.es
}

Recibido: 30/9/2019

Aceptado: 13/7/2020

Publicado: 29/1/2021

\section{Resumen}

Se presenta una investigación que busca determinar el impacto que tiene el uso de realidad virtual inmersiva (RVI) — asociada al desarrollo de actividades manipulativas y experienciales basadas en el enfoque STEM - en las actitudes científico-matemáticas de estudiantes de primero y segundo de ESO de un centro que se encuentra en un contexto en riesgo de exclusión social. Por medio de un procedimiento metodológico mixto cuantitativo-cualitativo, se han aplicado una serie de instrumentos a una muestra de 17 estudiantes y 1 docente. Los resultados indican la existencia de variaciones significativas, junto con un tamaño del efecto (TE) medio, en las actitudes hacia el aprendizaje de las ciencias $(p=0,000 ; \mathrm{TE}=0,535)$, no así en el ámbito de las matemáticas $(p=0,887 ; \mathrm{TE}=0,070)$. Asimismo, existen mejoras significativas en la autopercepción del aprendizaje de los contenidos STEM abordados en la propuesta $(p=0,000 ; \mathrm{TE}=0,944)$, valoración positiva del proyecto, tanto por parte de los estudiantes $(9,9$ sobre 10) como del profesor de las asignaturas de ciencias y matemáticas del centro (entrevista semiestructurada). Se concluye que la inclusión de este tipo de tecnologías impacta positivamente en las actitudes científico-matemáticas del estudiantado participante.

Palabras clave: realidad virtual; actitud hacia las ciencias; actitud hacia las matemáticas; exclusión social; educación STEM

* Agradecemos al CDP Escolapios-Cartuja, estudiantado, profesorado y dirección, su inestimable participación, colaboración y compromiso durante todo el desarrollo de esta investigación. Además, han sido, sin duda, estímulo e inspiración para los autores de este documento. 
Resum. Ús de les tecnologies immersives i el seu impacte en les actituds cientifico-matemàtiques dels estudiants d'educació secundària obligatòria en un context en risc d'exclusió social

S'hi presenta una investigació que té com a objectiu determinar l'impacte de l'ús de la realitat virtual immersiva (RVI) —associada amb activitats de manipulació $\mathrm{i}$ experiència basades en l'enfocament STEM - en les actituds científico-matemàtiques d'estudiants de primer i segon d'ESO d'un centre ubicat en un context d'exclusió social severa. Per mitjà d'un procediment metodològic mixt quantitativo-qualitatiu, s'han aplicat diferents instruments a una mostra de 17 estudiants i 1 docent. Els resultats indiquen l'existència de variacions significatives, juntament amb una mida de l'efecte (TE) mitjà, en les actituds cap a l'aprenentatge de les ciències $(p=0,000 ; \mathrm{TE}=0,535)$, però no gens en l'àmbit de les matemàtiques $(p=0,887 ; \mathrm{TE}=0,070)$. Així mateix, hi ha millores significatives en l'autopercepció de l'aprenentatge dels continguts STEM abordats en la proposta $(p=0,000$; $\mathrm{TE}=0,944)$, valoració positiva del projecte, tant per part dels estudiants $(9,9$ sobre 10$)$ com del professor de les assignatures de ciències i matemàtiques del centre (entrevista semi). $S^{\prime}$ hi conclou que la inclusió d'aquest tipus de tecnologies impacta positivament en les actituds científico-matemàtiques de l'estudiantat participant.

Paraules clau: perfil de professor; formació professional docent; anglès com a llengua estrangera; ALE; Xile

Abstract. Use of Immersive Technologies and their impact on the scientific-mathematical
attitudes of Secondary Education students in a context of risk of social exclusion

Research is presented that aims to determine the impact of the use of Immersive Virtual Reality (IVR), associated with manipulative/experiential activities based on the STEM approach, on the scientific-mathematical attitudes of 1st and 2nd year E.S.O. students, in a center located in a context of severe social exclusion. Through a mixed quantitativequalitative methodological procedure, different instruments have been applied to a sample of 17 students and 1 teacher. The results indicate the existence of significant variations, together with an average effect size (ES), in attitudes towards learning science $(\mathrm{p}=, 000$; $\mathrm{ES}=, 535)$, but not in the field of mathematics $(\mathrm{p}=, 887$; $\mathrm{ES}=, 070)$. Likewise, there are significant improvements in the self-perception of learning the STEM contents addressed in the proposal $(\mathrm{p}=, 000 ; \mathrm{ES}=, 944)$, a positive evaluation of the project by both students (9.9 out of 10) and by the teacher of the center's Science and Mathematics subjects (semistructured interview). It is concluded that the inclusion of this type of technologies positively impacts the scientific-mathematical attitudes of participating students.

Keywords: virtual reality; attitudes toward sciences; attitudes toward mathematics; socially disadvantaged; STEM education

\section{Sumario}

$\begin{aligned} \text { 1. Introducción, } & \text { 3. Resultados } \\ \text { fundamentación y objetivos } & \text { 4. Discusión y conclusiones } \\ \text { 2. Método } & \text { Referencias bibliográficas }\end{aligned}$




\section{Introducción, fundamentación y objetivos}

Frente a la escasa motivación existente por parte de estudiantes de educación secundaria, especialmente en lo referente al área científica (Organización para la Cooperación y el Desarrollo Económico, 2015; Rocard, 2007), se propone fomentar la diversificación de estrategias para la enseñanza de las ciencias buscando, entre otros aspectos, mejorar las actitudes del estudiantado e incentivar el aprendizaje de dicha área (Confederación de Sociedades Científicas de España, 2011). Así, el enfoque STEM ${ }^{1}$ apunta hacia el desarrollo de habilidades científico-matemáticas desde una perspectiva aplicada e integrada, promoviendo con fuerza la incorporación de la tecnología en la enseñanza. Sin embargo, la implementación de esta educación STEM resulta problemática y son pocos los proyectos que cumplen con la visión integrada y articulada de las disciplinas y, por lo general, la integración se realiza de forma parcial (Martín Páez et al., 2019).

En el informe Horizon Report: K-12 Edition (Freeman et al., 2017) se presenta una panorámica acerca de cómo la realidad aumentada (RA), la realidad virtual inmersiva (RVI), la robótica y el movimiento Maker se están incorporando al ámbito educativo. Se trata de tecnologías emergentes en educación, para cuyo uso más efectivo se requiere una exhaustiva investigación educativa que todavía es incipiente (Makokha, 2017; Ferrada, Díaz-Levicoy, Carrillo-Rosúa y Silva-Díaz, 2020; Raposo-Rivas y Martínez-Figueira, 2019), pero con gran potencial para el aprendizaje (Badeleh, 2019; Caballero-García y Fernández, 2019).

La búsqueda de estrategias para mejorar la actitud hacia las ciencias y las matemáticas son más acuciantes en contextos de exclusión social, determinados por factores generalmente colectivos que afectan a las diferencias de acceso a contenidos, experiencias y recursos, lo que impacta en el proceso educativo del estudiantado y propicia, de forma negativa, un posible fracaso o abandono escolar (Tarabini, 2017). Más aún, la escasez de estudiantes que continúan con estudios universitarios en el ámbito STEM es apreciablemente mayor.

\subsection{Actitudes hacia las ciencias y las matemáticas}

Las actitudes hacia las ciencias han sido foco de numerosas investigaciones en educación (Aguilera y Perales-Palacios, 2018; Osborne, Simon y Collins, 2003) y se presentan de modo multidimensional, siendo factores relevantes los siguientes (Zhang y Campbell, 2011): la percepción del profesor de ciencias, la ansiedad, la valorización de las ciencias, la autoestima en las ciencias,

1. STEM: Acrónimo de Science, Tecnology, Engineering, and Mathematics. Hace referencia a una reciente tendencia educativa nacida en EE. UU. que implica, entre otras orientaciones, un enfoque de la enseñanza interdisciplinaria en relación con estas áreas de conocimiento, cambio en la finalidad de la educación científica y un refuerzo de metodologías didácticas más dinámicas. 
la motivación hacia las ciencias, la satisfacción o el disfrute de la ciencia y las actitudes de otros (compañeros o amigos) hacia dicha disciplina. Asimismo, corresponden a elementos implícitos al ser humano que incluso inconscientemente desempeñan un papel esencial en todos los aprendizajes (Pozo y Gómez, 1998), viéndose influenciadas por el contexto (Schwarz, 2007) y los elementos culturales (Osborne et al., 2003).

Existe una relación entre las actitudes y el logro académico frecuentemente asociadas a experiencias de la infancia (Osborne et al., 2003), que pueden ser positivas o negativas, dependiendo de las creencias de los propios estudiantes respecto a sus capacidades académicas (Zan y Di Martino, 2007) o de la vinculación emocional que se tenga con la asignatura (Mata et al., 2012). Asimismo, influyen en la disposición a vincularse con temas relacionados con la ciencia y, además, constituyen un elemento favorecedor de la alfabetización científica (Bybee y McCrae, 2011).

\subsection{Realidad virtual inmersiva en educación}

La tecnología educativa ha presentado un crecimiento acelerado durante los últimos años (Cabero, 2015). Su integración didáctica en el proceso de ensenanza ha sido de gran utilidad, especialmente en asignaturas de tipo científico (Makokha, 2017), aunque su explosiva inclusión en el ámbito escolar ha traído consigo una serie de dificultades, entre ellas la capacidad de los centros para absorberlas (Cabero, 2015).

Según Cipresso et al. (2018), la RVI implica tres ideas clave:

a) Inmersión: cantidad de sentidos estimulados en el entorno RVI. El grado de inmersión puede variar según el diseño del entorno RVI y el uso de sensores adicionales (p. ej. auriculares —oído—, sensores hápticos - tacto-, entre otros).

b) Presencia en un entorno RVI: se relaciona con el nivel de realismo del diseño del entorno virtual y la posibilidad de «interactuar» en él.

c) Interacción con el entorno: cuestión relacionada con las expectativas de realidad del usuario y las propuestas en el entorno de RVI. A mayor cercanía con la realidad, mayor sensación de presencia y, por consiguiente, mayor interacción.

La RVI facilita que el estudiante comprenda mejor los contenidos, lo que aumenta su motivación por aprender (Truchly et al., 2018); permite obtener un sentido del espacio 3D del que carece cualquier otro sistema de representación gráfica, lo que favorece la explicación de conceptos complejos o abstractos (Vera, Ortega y Burgos, 2003); además, ayuda a mejorar habilidades de visualización espacial, especialmente en el aprendizaje de la geometría en tres dimensiones (3D) (Gwee, 2014). El uso de la RVI en la educación es muy reciente (Freeman et al., 2017), se trata, entonces, de una muy nueva fuente de información cuya investigación es muy incipiente. 


\subsection{Educación STEM}

El surgimiento del «movimiento» STEM tiene sus orígenes en la década de 1990 en Estados Unidos (Sanders, 2009; Bybee, 2013). Aun cuando el término STEM desde entonces ha sido ampliamente utilizado (Bybee, 2013), incluso hasta el punto de "comercializarse» (Sanders, 2009), no existe una clara definición de él. Bybee (2013) manifiesta que se requiere de un contexto que delimite su significado, y Sanders (2009), que se debe, al menos, articular dos o más disciplinas y no necesariamente las cuatro de forma simultánea, pudiéndose relacionar una asignatura STEM con otra asignatura escolar de otra índole (p. ej. historia, ciencias sociales, artes, entre otras). Además, Martín-Páez et al. (2019) sintetizan que la articulación de las disciplinas debe ir acompañada de una metodología que tenga la capacidad de potenciar la integración (p. ej. las metodologías de indagación o el aprendizaje basado en proyectos).

\subsection{Contextos educativos de exclusión social}

El concepto de exclusión social se encuentra muy relacionado con aspectos vinculados a la ciudadanía social (p. ej. trabajo, salud, educación, vivienda, calidad de vida, entre otros), siendo el sector excluido el que se encuentra al margen de la obtención de ciertos derechos laborales, culturales y/o escolares (Jiménez, 2008). Además, corresponde a un proceso amplio y gradual, en el que el estudiantado, a modo general, se ve expuesto, sin distinción, a espacios de vulnerabilidad, en los que su proceso de escolarización sufre un riesgo potencial, donde el origen socioeconómico del estudiantado influye, en gran medida, en el fenómeno de la exclusión social, principalmente porque se trata de hogares que poseen bajo capital cultural y, por tanto, proyectan en sus hijos las mismas carencias (Tarabini, 2017), lo que afecta a las creencias y/o a las actitudes sobre estos (Gorard y See, 2009). Elementos como el desconocimiento del español por parte de los familiares de los escolares, circunstancia que puede ser común en estos contextos, agravan más aún la brecha que favorece el fracaso escolar (Escarbajal et al., 2019).

Frente a este fenómeno, Jiménez (2012) manifiesta la importancia de fomentar la colaboración entre las escuelas y la comunidad. Uno de estos espacios de colaboración es conocido como comunidades de aprendizaje, constituye el contexto de esta investigación e implica también potenciar las interacciones de los estudiantes y la extensión del aprendizaje a los espacios de la tarde (Flecha y Puigvert, 2002).

\subsection{Objetivos}

En esta investigación, que implica el diseño y la implementación de una propuesta STEM orientada a potenciar las actitudes científico-matemáticas en estudiantes de primero y segundo de Enseñanza Secundaria Obligatoria (ESO) en un contexto de exclusión social, se plantean los siguientes objetivos: 
a) Comprobar si la intervención didáctica mejora las actitudes de los estudiantes participantes en referencia a las matemáticas y las ciencias.

b) Considerar la valoración de los aprendizajes por parte del estudiantado respecto a los contenidos STEM.

c) Conocer la valoración que hace el estudiantado respecto del uso de la RVI.

d) Caracterizar las dificultades y los puntos fuertes del uso de la RVI y sus posibilidades de integración con actividades manipulativas y experienciales en el ámbito STEM en contextos de exclusión social severa, por medio de la valoración del profesorado respecto al proyecto.

\section{Método}

La investigación responde a un enfoque mixto, cuantitativo y con un diseño cuasiexperimental con un solo grupo (León y Montero, 2015), con aplicación de un pretest y de un postest para la medición de actitudes y solo de un postest para la autovaloración de aprendizajes y del proyecto en sí. Además, cuenta con una fase cualitativa basada en la aplicación de una entrevista semiestructurada tras la intervención y una encuesta de satisfacción de la propuesta. La elección de este tipo de diseño se basa en la complementación señalada por Hernández, Fernández y Baptista (2014), dada la necesidad de comprender en profundidad los resultados de la investigación.

\subsection{Población y participantes}

La investigación se ha desarrollado en un centro educativo concertado que integra el proyecto educativo llamado comunidades de aprendizaje, ubicado en una zona socialmente muy vulnerable de la ciudad de Granada. La población está conformada por estudiantes de primero y segundo de ESO.

Los participantes (grupo experimental) han sido seleccionados mediante un muestreo no probabilístico intencional (Cardona, 2002), siguiendo criterios de accesibilidad a la muestra y de interés por participar en la investigación. Se trata de 17 estudiantes (de 12 y 13 años), de los cuales 10 son mujeres ( 7 de primero de ESO y 3 de segundo de ESO) y 7 son varones (4 de primero de ESO y 3 de segundo de ESO). El resto de los dos grupos clase (26 estudiantes) decidió no participar en la intervención o no pudo hacerlo por incompatibilidad de horario, aunque sí intervino en la medición de dos de los instrumentos, con lo que conformó una medida de control en las variables implicadas. Además, en la vía cualitativa participó como informante el profesor del estudiantado de las asignaturas científico-matemáticas.

\subsection{Instrumentos de recogida de información}

Los cuestionarios The Psychometric Evaluation of a Three-Dimension Elementary Science Attitude Survey (TDSAS), de Zhang y Campbell (2011), y la escala de actitudes hacia las matemáticas (EAM), propuesta por Palacios et 
al. (2014), han sido utilizados para determinar la actitud de los estudiantes hacia las ciencias y las matemáticas, respectivamente, y ambos han sido valorados mediante una escala de Likert de 1 a 5. Adicionalmente, se ha diseñado, ad hoc para este estudio, un cuestionario tipo Knowledge and Prior Study Inventory (KPSI, Young y Tamir, 1977), que tiene por finalidad la autovaloración de los aprendizajes conceptuales asociados a los contenidos desarrollados durante el proyecto. El cuestionario cuenta con 24 ítems distribuidos en cuatro dimensiones STEM. Se utiliza una escala de Likert de 1 a 4 para valorar lo que los alumnos sabían antes ( $p r e$ ) y lo que consideran que saben ahora (post), de acuerdo con los valores siguientes: 1 = Lo sabía $/$ No sé nada; 2 = Lo sabía / Sé poco; 3 = Lo sabía / Lo sé bien, y 4 = Lo sabía / Lo sé muy bien, puedo explicarlo a otros.

Se ha realizado el cálculo de Alfa de Cronbach de estos tres instrumentos y se han registrado valores similares a los originales: TDSAS y EAM $(\alpha=0,92$ pre y post), KPSI ( $\alpha=0,92$ pre y $\alpha=0,87$ post). Del Rincón et al. (1995, p. 68) señalan que «una fiabilidad mínima de 0,70 es considerada aceptable para la toma de decisiones».

Se ha elaborado una encuesta de satisfacción ad hoc con la finalidad de que los participantes valoren la experiencia. La encuesta es de tipo mixto, con 17 preguntas de la escala de Likert del 1 al 10 distribuidas en: a) organización (4), b) STEM + VR (4), c) actividades manipulativas y experienciales (4), d) materiales utilizados (3) y $e$ ) valoración general (2). Adicionalmente cuenta con preguntas abiertas dirigidas a caracterizar la valoración del estudiantado sobre el proyecto y a recoger comentarios y/o sugerencias.

Por último, se ha diseñado una entrevista semiestructurada de cuatro dimensiones (tabla 1) que permite caracterizar aspectos actitudinales de los estudiantes que participaron en el proyecto a través de la percepción de su profesor del ámbito científico-matemático.

Tabla 1. Dimensiones de la entrevista semiestructurada

\begin{tabular}{ll}
\hline 1. Perfil profesional. & $\begin{array}{l}\text { Se describe el perfil profesional del docente y la relación existente } \\
\text { con los estudiantes. }\end{array}$ \\
$\begin{array}{l}\text { 2. Utilidad de tecnologías } \\
\text { emergentes en la educación } \\
\text { (TEE) y actividades basadas } \\
\text { en un enfoque STEM. }\end{array}$ & $\begin{array}{l}\text { Se busca trazar una panorámica sobre la utilidad que podría } \\
\text { representar la inclusión de herramientas tecnológicas en la ense- } \\
\text { ñanza, principalmente el uso de RVI en la enseñanza de materias } \\
\text { científo-matemáticas y la realización de actividades manipula- } \\
\text { tivas y experimentales que consideren un enfoque articulador de } \\
\text { disciplinas (STEM). }\end{array}$ \\
$\begin{array}{l}\text { 3. Actitudes hacia las cien- } \\
\text { cias y las matemáticas. }\end{array}$ & $\begin{array}{l}\text { Se pretende obtener una visión, por parte del profesor, respecto a } \\
\text { la actitud, o el cambio de actitud, que manifiestan los estudiantes } \\
\text { que participaron en el proyecto respecto al aprendizaje de las } \\
\text { ciencias y las matemáticas. }\end{array}$ \\
$\begin{array}{l}\text { 4. Relación entre actitudes y y busca determinar si la participación en el proyecto de RVI por } \\
\text { rendimiento. }\end{array}$ & $\begin{array}{l}\text { Se barte del estudiantado ha tenido efectos positivos en la actitud } \\
\text { partian } \\
\text { hacia el aprendizaje de asignaturas científico-matemáticas. }\end{array}$ \\
\hline
\end{tabular}

Fuente: elaboración propia. 


\subsection{Propuesta didáctica}

Se ha diseñado una propuesta didáctica (Silva-Díaz, Carrillo-Rosúa y Fernández-Plaza, 2019) que articula el uso del software STEM + VR de tecnología RVI con cuatro actividades manipulativas y experienciales, a realizar mediante trabajo colaborativo y que impliquen el uso de materiales tecnológicos. Adicionalmente, se contempla un tiempo de refuerzo académico. Se contextualizan en diversas situaciones problemáticas siguiendo el modelo de propuestas STEM como las de Fernández et al. (2020), bajo la temática común de un viaje espacial abordando los ámbitos STEM de forma integrada (Sanders, 2009), así como una orientación indagatoria y competencial (Organización para la Cooperación y el Desarrollo Económico, 2015). Implica cuatro sesiones de 105 minutos, con una experiencia de siete horas por grupo. Dos grupos de tres participantes intervienen en cada sesión. En la figura 1 se presenta la distribución de actividades por disciplina STEM.

\subsection{Procedimiento}

La implementación de la propuesta se coordinó con el docente de las asignaturas científico-matemáticas de los participantes, quien, además, aplicó los cuestionarios TDSAS y EAM antes y después de la intervención en los dos grupos clase en su totalidad. Además, los investigadores aplicaron, postinter-

Figura 1. Distribución de las sesiones en función de la disciplina STEM trabajada. La experiencia $\mathrm{RVI}$ y el refuerzo académico se realizan de forma paralela y los grupos alternan su participación en cada una de ellas

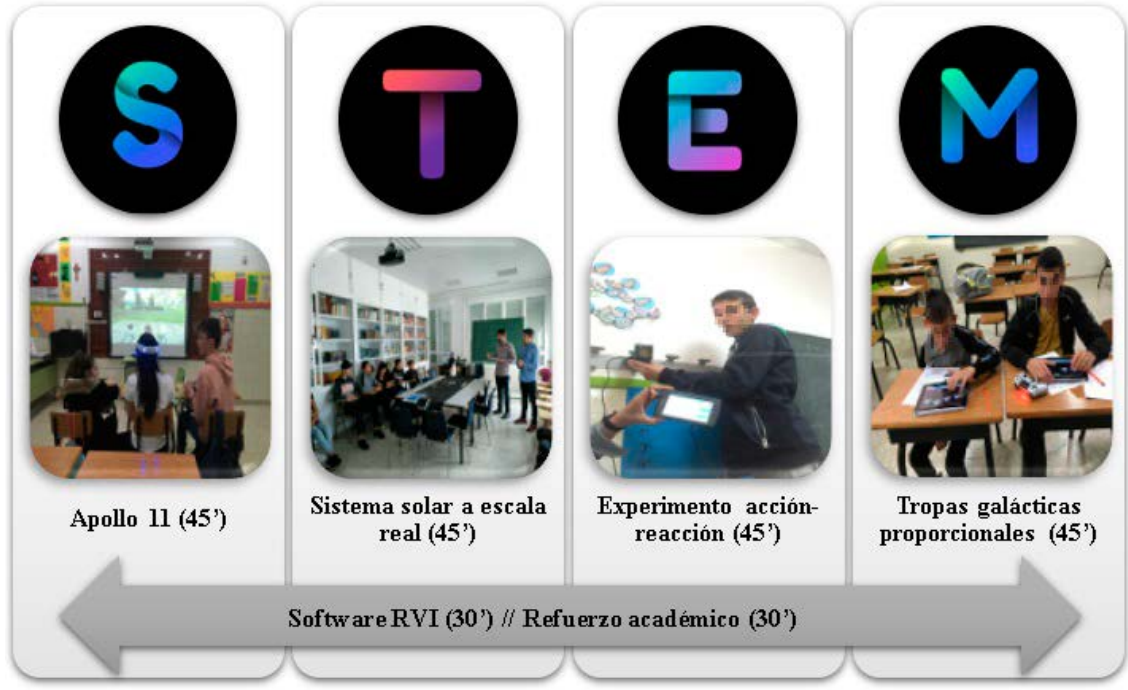

Fuente: elaboración propia. 
vención, la encuesta de satisfacción y el cuestionario KPSI a los participantes y la entrevista al profesorado implicado. La investigación concluye con una ceremonia de celebración donde los estudiantes participantes (grupo experimental) son reconocidos con un diploma.

\subsection{Tratamiento de los datos}

Para el tratamiento cuantitativo de los datos se utilizan los programas SPSS v25 y STATA v15.1, mientras que para el análisis cualitativo se ha empleado el MAXQDA v12.

Se descartan los criterios de normalidad y se aplican las pruebas de Wilcoxon y el tamaño del efecto (TE) al grupo experimental.

El análisis cualitativo se desarrolla mediante categorización mixta (León y Montero, 2015) deductivo-inductiva aplicada a la encuesta de satisfacción (tabla 2) y a la entrevista semiestructurada al profesorado (tabla 3).

Tabla 2. Codificación de categorías de análisis para la encuesta de satisfacción

\begin{tabular}{|c|c|}
\hline Categoría & Subcategorías \\
\hline 1. Implementación de la propuesta didáctica. & $\begin{array}{l}\text { a) Organización. } \\
\text { b) Horario del proyecto. } \\
\text { c) Duración del proyecto. } \\
\text { d) Cantidad de participantes. }\end{array}$ \\
\hline $\begin{array}{l}\text { 2. Utilidad de tecnologías emergentes en la } \\
\text { educación (TEE). }\end{array}$ & $\begin{array}{l}\text { a) Gafas de realidad virtual inmersiva (RVI) } \\
\text { b) STEM + VR. } \\
\text { c) Consola PlayStation. } \\
\text { d) Realidad virtual. }\end{array}$ \\
\hline $\begin{array}{l}\text { 3. Valoración de las actividades propuestas } \\
\text { basadas en un enfoque STEM. }\end{array}$ & $\begin{array}{l}\text { a) Apollo } 11 . \\
\text { b) Sistema solar a escala. } \\
\text { c) Experimento acción-reacción (globos). } \\
\text { d) Tropas proporcionales galácticas. } \\
\text { e) Trabajo colaborativo. }\end{array}$ \\
\hline 4. Elementos sociales y personales. & $\begin{array}{l}\text { a) Compartir con los amigos. } \\
\text { b) Diversión. } \\
\text { c) Jugar. }\end{array}$ \\
\hline
\end{tabular}

Fuente: elaboración propia.

Tabla 3. Codificación de categorías de análisis para la entrevista al profesorado

\begin{tabular}{|c|c|}
\hline Categoría & Subcategorías \\
\hline 1. Perfil profesional. & $\begin{array}{l}\text { a) Datos personales. } \\
\text { b) Trayectoria profesional. } \\
\text { c) Asignaturas que imparte. } \\
\text { d) Relación con los participantes. }\end{array}$ \\
\hline 2. Implementación de la propuesta didáctica. & $\begin{array}{l}\text { a) Organización. } \\
\text { b) Horario del proyecto. } \\
\text { c) Valoración del proyecto. } \\
\text { d) Cantidad de participantes. }\end{array}$ \\
\hline
\end{tabular}




$\begin{array}{ll}\begin{array}{l}\text { 3. Utilidad de tecnologías emergentes en la } \\ \text { educación (TEE) y actividades basadas en }\end{array} & \begin{array}{l}\text { b) Realidad virtual. } \\ \text { c) Tecnologías en educación. } \\ \text { un enfoque STEM. }\end{array} \\ \begin{array}{ll}\text { d) Enfoque STEM de enseñanza. } \\ \text { e) Rol del profesorado. }\end{array} \\ \begin{array}{ll}\text { 4. Actitudes de los participantes hacia las } & \text { a) Percepción del estudiantado sobre el proyecto. } \\ \text { ciencias y las matemáticas. } & \text { b) Disposición o actitud en sus clases. } \\ & \text { c) Cambios positivos de actitud en sus clases. } \\ & \begin{array}{l}\text { d) Mayor participación en sus clases. } \\ \text { e) Opinión de los participantes sobre el proyecto. }\end{array} \\ & \text { f) Relación entre actitudes y rendimiento. }\end{array} \\ \begin{array}{ll}\text { a) Relaciones personales entre el estudiantado. } \\ \text { b) Diferencias sociales. }\end{array} \\ \begin{array}{ll}\text { c) Brecha sociocultural. } \\ \text { d) Recursos tecnológicos y/o económicos. } \\ \text { e) Características del centro. }\end{array}\end{array}$

Fuente: elaboración propia.

\section{Resultados}

\subsection{Actitud hacia las ciencias y las matemáticas}

En las figuras 2 y 3 se presentan, respectivamente, los resultados medios disgregados por dimensiones, del pretest y del postest de actitud hacia las ciencias y actitud hacia las matemáticas. Asimismo, en ambas figuras se presentan las puntuaciones medias del total de la población a modo referencial.

Se registran variaciones positivas pretest y postest en las puntuaciones medias de todas las dimensiones del cuestionario TDSAS:

I. Los estudiantes están conectados afectivamente con las ciencias $(+0,34)$.

II. Los estudiantes aprecian la importancia de la ciencia y la investigación científica para la sociedad $(+0,26)$.

III. Los estudiantes están involucrados en conductas reales de aprendizaje de la ciencia $(+0,31)$, siendo significativas y con una valoración del tamaño del efecto medio-alto $(\mathrm{TE}=0,495,0,425$ y 0,614$)$. En cuanto a las actitudes hacia las ciencias, en general se registran variaciones positivas $(+0,3)$ de carácter estadísticamente significativo y, además, se evidencia la efectividad del programa en un rango medio $(\mathrm{TE}=0,535)$. En todos los casos, estas variaciones positivas son más amplias que en el conjunto de la población.

En cuanto a las actitudes hacia las matemáticas, las dimensiones percepción de la incompetencia matemática (I) y percepción de la utilidad (III) muestran puntuaciones que reflejan mejoría $(+0,17$ y $+0,23$, respectivamente). Sin embargo, dichas diferencias no son estadísticamente significativas y los valores del tamaño del efecto indican que tampoco se puede asegurar la efectividad de la intervención en lo que respecta a estas dimensiones $(\mathrm{TE}=0,190$ y 0,391). En cuanto a las otras dimensiones, los resultados van en la misma línea, arro- 
Figura 2. Resultados del cuestionario TDSAS agrupados por dimensiones
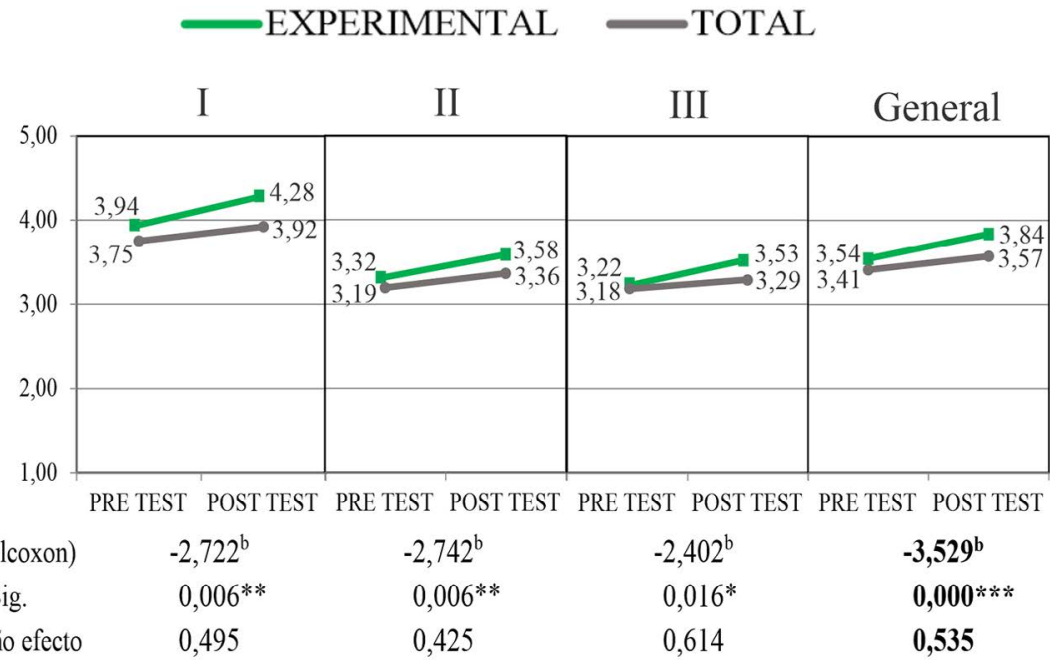

Estadísticamente significativo al ${ }^{*} p<0,05{ }^{* *} p<0,01{ }^{* * *} p<0,001$. Cohen (1988) establece los siguientes valores de interpretación para el tamaño del efecto (TE): bajo $<0,2$; medio entre 0,2 y 0,5 y alto $>0,8$.

Fuente: elaboración propia.

Figura 3. Resultados del cuestionario EAM agrupados por dimensiones

-EXPERIMENTAL TOTAL

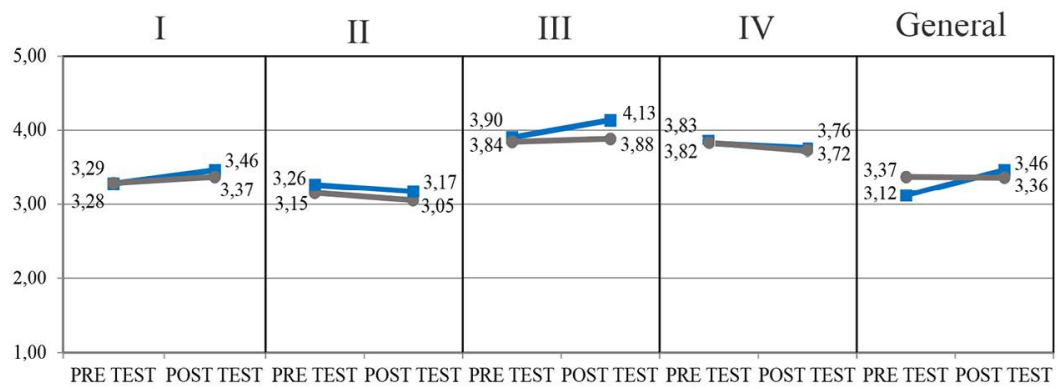

$\begin{array}{cccccc}\text { (Wilcoxon) } & -0,910^{\mathrm{b}} & -0,622^{\mathrm{c}} & -1,159^{\mathrm{b}} & -0,862^{\mathrm{c}} & \mathbf{- 0 , 1 4 2}^{\mathrm{b}} \\ \text { Sig. } & 0,363 & 0,534 & 0,247 & 0,388 & \mathbf{0 , 8 8 7} \\ \text { maño efecto } & 0,190 & -0,110 & 0,391 & -0,107 & \mathbf{0 , 0 7 0}\end{array}$

Fuente: elaboración propia.

jando incluso diferencias negativas. Cuando se realiza una valoración general de las actitudes hacia las matemáticas, se observa una leve variación positiva $(+0,34)$ no estadísticamente significativa y que, según la clasificación de 
Cohen, no logra asegurar la efectividad de la intervención $(\mathrm{TE}=0,070)$. No obstante, si consideramos el total de la población, la diferencia pretest y postest es incluso negativa $(-0,01)$.

\subsection{Autopercepción de los aprendizajes}

En cuanto a la autovaloración de los aprendizajes relacionados con los contenidos trabajados en la intervención, se presentan los resultados agrupados en las dimensiones STEM y la totalidad del instrumento (figura 4).

Se presentan variaciones positivas en todas las dimensiones del instrumento. Los mejores resultados se obtienen en ingeniería $(+0,58)$, los de ciencias y tecnología son intermedios $(+0,53 \mathrm{y}+0,50)$, mientras que matemáticas registra la menor variación $(+0,37)$. En todos los casos, las diferencias son estadísticamente significativas.

Figura 4. Resultados del test KPSI agrupados por dimensiones

\section{EXPERIMENTAL}

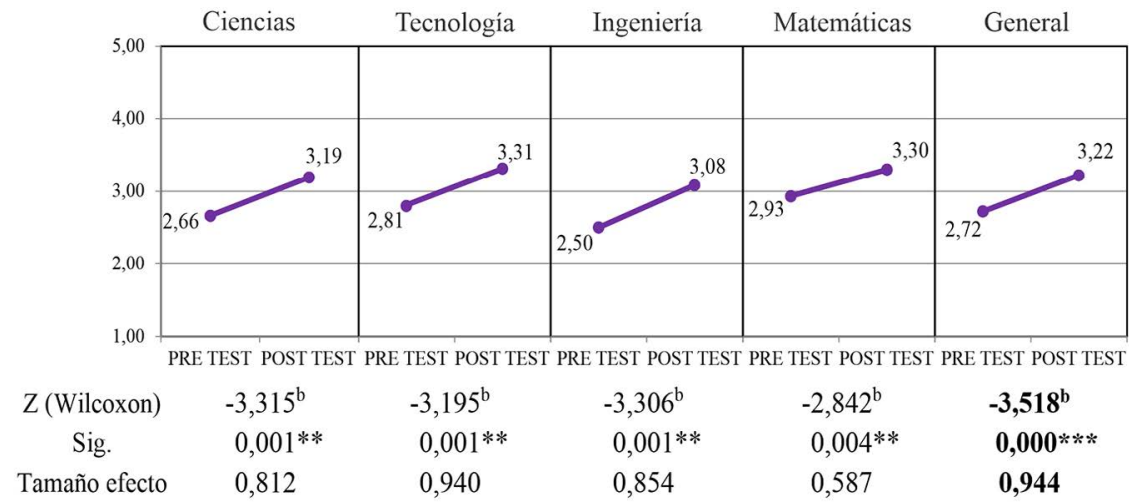

Estadísticamente significativo al ${ }^{*} p<0,05{ }^{* *} p<0,01{ }^{* *} p<0,001$

Fuente: elaboración propia.

\subsection{Satisfacción respecto al programa por parte del estudiantado}

En lo referente a la satisfacción en referencia al proyecto, en la figura 5 se presentan los resultados cuantitativos (figura 5) y en la tabla 4, los cualitativos.

Se realiza una valoración positiva del proyecto en términos generales $(9,9$ sobre 10), mientras que por dimensiones se aprecian resultados muy similares en las actividades $(9,48)$ y en los materiales utilizados, por ejemplo, la RVI $(9,75)$. Las dimensiones con menor valoración corresponden a la organización $(9,0)$, que implica el horario del proyecto y el software STEM + VR $(8,75)$, principalmente por aspectos técnicos como el audio. 
Figura 5. Se presentan puntuaciones mínimas, máximas y medias, junto con valores atípicos por dimensión

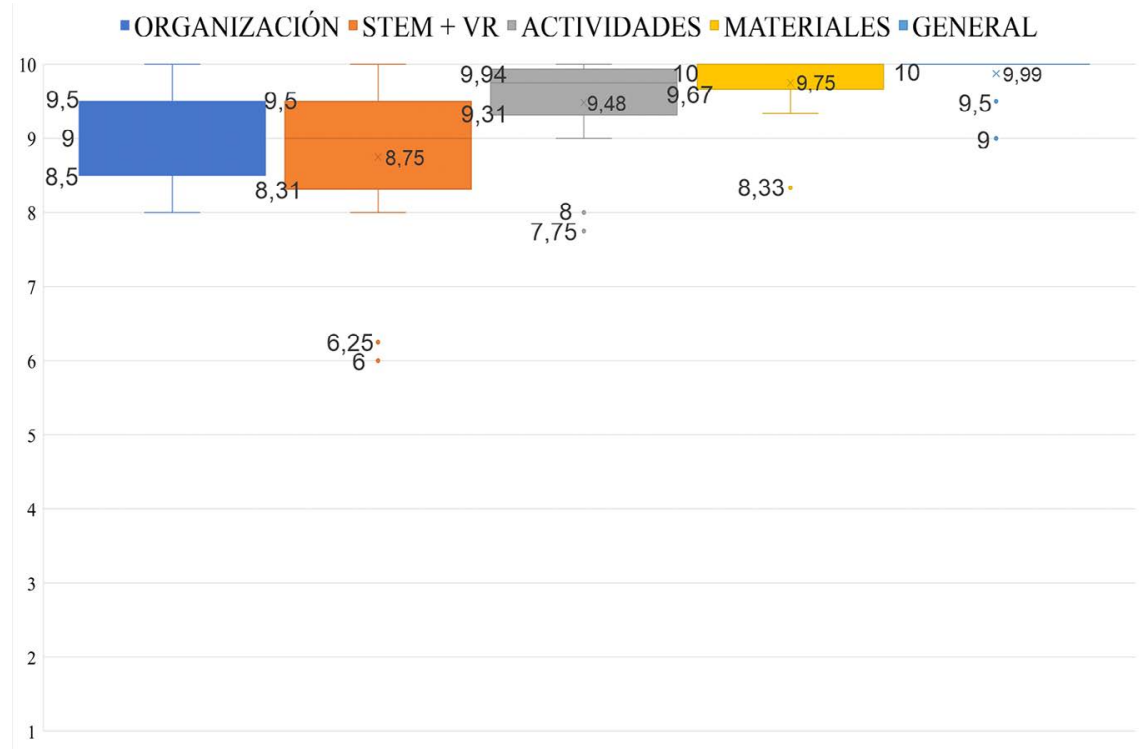

Fuente: elaboración propia.

Tabla 4. Resultados del análisis de la encuesta de satisfacción

\begin{tabular}{|c|c|c|c|c|c|c|c|c|c|c|c|c|c|c|c|c|}
\hline & \multicolumn{4}{|c|}{$\begin{array}{c}\text { Propuesta } \\
\text { didáctica (1) }\end{array}$} & \multicolumn{4}{|c|}{$\begin{array}{l}\text { Utilidad } \\
\text { TEE (2) }\end{array}$} & \multicolumn{5}{|c|}{$\begin{array}{l}\text { Actividades } \\
\text { STEM (3) }\end{array}$} & \multicolumn{3}{|c|}{$\begin{array}{l}\text { Elementos } \\
\text { sociales (4) }\end{array}$} \\
\hline & $a$ & $b$ & $c$ & $d$ & $a$ & $b$ & $c$ & $d$ & $a$ & $b$ & $c$ & $d$ & $e$ & $a$ & $b$ & $c$ \\
\hline $\begin{array}{l}\text { Frecuencia } \\
\text { subcategoría }\end{array}$ & 5 & 0 & 3 & 1 & 8 & 6 & 3 & 7 & 1 & 2 & 5 & 4 & 2 & 4 & 7 & 4 \\
\hline $\begin{array}{l}\text { Frecuencia } \\
\text { categoría }\end{array}$ & & $\varsigma$ & 9 & & & 24 & & & & & 14 & & & & 15 & \\
\hline
\end{tabular}

Fuente: elaboración propia.

Las valoraciones cualitativas refrendan los resultados cuantitativos. Aun cuando los estudiantes contestan de forma escueta y carente de argumentación, se observa que la categoría utilidad de las TEE (2) ha sido la que ha tenido una mayor frecuencia (tabla 4) y la que siempre ha recibido una valoración positiva. $\mathrm{Al}$ ser consultados por los dos aspectos que más les gustaron del proyecto, responden: «La PS4», «La VR» y «Las gafas de realidad virtual».

En cuanto a las actividades STEM (3) destacan positivamente su realización. Consideramos que esta situación se debe, en parte, al contexto informal en el que se desarrollan y la interacción con sus compañeros en la realización de actividades desafiantes: «Las gafas y el experimento de globos», "Apollo 11» $\mathrm{y}$ «El globo» $\mathrm{o}$ «Las gafas de realidad virtual y el experimento acción-reacción». 
Respecto de la propuesta didáctica (1), los estudiantes han realizado una menor cantidad de comentarios (tabla 4). Dentro de sus opiniones en esta categoría, destacan las siguientes: «Es increíble y los monitores son excelentes», «Me gustaría que hubiese más proyectos». Asimismo, frente a la pregunta «¿Participarías de nuevo en una continuación del proyecto?», el 100\% de los encuestados responde que sí.

Otro aspecto relevante es la importancia que tiene para ellos desarrollar actividades mediante el juego. Estas observaciones han sido categorizadas como elementos sociales (4), donde destacan: «Nos divertimos y aprendemos a la vez», «Estar con la Play y jugar a esos juegos y estar con los amigos».

\subsection{Opinión del profesorado}

Se presentan aquí los resultados obtenidos al examinar la entrevista al profesor de ciencias y de matemáticas de los estudiantes participantes. En el análisis se han aplicado las categorías definidas en la tabla 1. En la figura 6 se presenta el peso de cada categoría en la entrevista junto con las relaciones entre estas.

Figura 6. Sistema de códigos de entrevista y peso en función de la frecuencia de registro, junto con la relación entre categorías. Obtenido con el software MAXQDA v.12

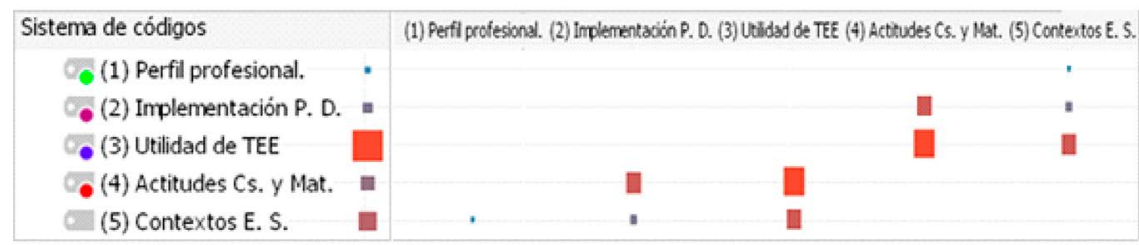

Fuente: elaboración propia.

Se aprecia la importancia que ha tenido la utilidad de las TEE y el enfoque STEM (3) para el profesor del centro:

Sí, bueno, ha sido útil en tener $[s i c]$ como un despertar. Haría falta más dosis..., con más contenidos... Claro que sí, pues... más gafas, que llegaran más... Entonces... eh..., pues es lo que te digo. No vamos a ser tan ilusos y decir... j*** tío [sic], está... Bueno, es una herramienta poderosa y ha ilusionado y en su dosis justa... y cada vez profundamente más... Podría llegar a..., pienso yo, podría llegar a hacer el efecto que tiene que hacer... De momento yo me quedo con que ha sido como un despertar.

Sin embargo, aún no está claro cómo utilizar estas tecnologías: «Que ocupe el lugar que tiene que ocupar. Yo creo que ahora no lo tiene, ahora mismo es una cosa motivadora y tiene que ocupar el sitio que tiene que ocupar». En ese sentido, señala que existe una necesidad y una demanda por parte del estudiantado: 
[...] entonces, sí, los niños perciben, es verdad. Es que hay un desfase grandísimo. Los niños están mucho más adelantados, todos controlan los móviles, todo es digital, todo va por ahí... En cierta manera, la sociedad como que les está diciendo: «Se educa así, pero vienes aquí y yo te sigo dando la misma ficha que me dieron a mí hace 15 años».

Por cuanto urge la incorporación efectiva de la tecnología en la educación: «Claro, ese salto... o sea, cómo es que... que llegue la tecnología de verdad a la educación, pero a la enésima potencia. No tener un PC, sino que es necesario sacarle sentido a esto», y agrega: «Es que no puede ser que sigamos dando materiales del siglo XIX en el siglo XXI».

Otro de los aspectos cruciales de esta cuestión es la importancia que tienen para el docente los contextos educativos de exclusión social (5), de modo que concibe la tecnología como un «arma de doble filo»: "A mí me da un poco de miedo que esta tecnología acabe siendo exclusiva o abunde más en otros contextos y ahonde las diferencias en el sistema educativo, que queremos hacer igualitario", y puntualiza que existen, y siguen existiendo, diferencias sociales: «Donde, por tener más medios, pues tengan más acceso, más recursos y entonces la brecha se ahonde». Sin embargo, comprende el impacto que genera el uso de este tipo de tecnologías: «Entonces no me gustaría que esto fuese una herramienta que marcara todavía más diferencias. Debería usarse más en estos contextos para posibilitar a esta gente tener las mismas herramientas o mejores», y luego añade: «Eh, aquí es donde tiene que estar... Aquí es donde hay que poner la carne en el asador, porque si queremos una sociedad... así, más justa, se tiene que partir de una acción igualitaria».

Finalmente, respecto de la utilidad específica de la RVI, el profesor no tiene dudas: «No es lo mismo ver o estudiar una célula en una foto, en blanco y negro, en una ficha repetitiva... que meterte en una célula y decir "oye, esto es esto y vale para esto" y "oye, mira lo que está fabricando, mira cómo funciona"”. El impacto que tiene para el profesor la RVI en la enseñanza de las ciencias es potentísimo: «Oye, y es verdad, cuando la ciencia pasa del libro a la realidad..., pues la ciencia se multiplica por $1.000 »$.

Respecto de las relaciones entre categorías (figura 6), se observa que existe una estrecha relación entre las actitudes hacia las ciencias y las matemáticas (4) y la utilidad de las TEE y el enfoque STEM. Para el profesor del centro la propuesta ha sido motivante: «Los niños estaban motivados, me comentaban por la mañana: "oye, pues qué chulo esto..., pues qué chulo esto otro..."”, destacando, entre otros aspectos, las actividades que, como señala el profesor, han sido un "despertar»: «Esta alumna de segundo que me ha venido, porque era una alumna que estaba especialmente enfocada a la asignatura de letras, y oye..., y vino muy entusiasmada porque con la bolita, no..., esta que hicisteis..., midiendo velocidades y movimiento...». No obstante, el impacto en las actitudes no ha sido igual en todas las asignaturas: «Más en algunas que en otras, por ejemplo, en ciencias a lo mejor sí... Vimos las células procariotas, eucariotas, y ahí sí había mayor interés... Luego en las mates..., pues ya no 
sé..., no soy tan consciente». Esta aseveración se alinea con los resultados cuantitativos de la investigación, donde ha habido mejora en la actitud hacia las ciencias, pero no así en lo que respecta a las matemáticas.

\section{Discusión y conclusiones}

Considerando los objetivos $a$ y $b$ se evidencia el impacto positivo que ha tenido la implementación de la propuesta didáctica en las actitudes hacia las ciencias, mientras que en las matemáticas no ha sido tal. Dichas variaciones se podrían atribuir al desarrollo de actividades manipulativo-experienciales basadas en un enfoque STEM, debido a que facilitan la conexión entre el conocimiento científico y las experiencias reales de aprendizaje (Martín et al., 2019; McDonald, 2016; Sevian et al., 2018). Probablemente, el menor impacto en las actitudes hacia las matemáticas podría ser atribuible a la ansiedad que genera en el alumnado el hecho de enfrentarse a dicha disciplina (Akin y Kurbanoglu, 2011; Chinn, 2012) y que los estudiantes interpreten las experiencias vividas como científicas, aunque impliquen el empleo de habilidades matemáticas (p. ej. experimentos y cálculos asociados) que les motiven a aprender ciencias (Aguilera y Perales-Palacios, 2018; Cremin et al., 2015; Osborne et al., 2003), pero no las han percibido también como ejemplos de modelizaciones matemáticas del mundo. La misma situación ocurre a la hora de valorar los aprendizajes. Aunque los estudiantes manifiestan aprender en todas las dimensiones STEM, las matemáticas han sido las que registran menor variación. Además, al considerar la autovaloración (self-assessment) como un instrumento de valoración tan efectivo como la evaluación del profesor (Chang, Tseng y Lou, 2012), nos hace presumir que una propuesta de este tipo podría tener efectos positivos no solo en las actitudes, sino también en el rendimiento académico, siendo objeto de investigaciones en un futuro.

Por tanto, se podría concluir que, para mejorar la actitud hacia las matemáticas, se requiere un esfuerzo adicional para intentar que el estudiante haga nítidamente explícito el papel de dicha materia en la interpretación y la manipulación que se realiza a través de la ciencia de la realidad.

Respecto de los objetivos $c$ y $d$, el uso de la tecnología de RVI ha sido positivamente valorada como una herramienta para la adquisición de conocimientos, principalmente porque propone un aprendizaje basado en la experimentación. Esta es una de las principales potencialidades de la realidad virtual en la educación (Freeman et al., 2017; Radianti et al., 2019; Truchly et al., 2018). Y es que el uso de herramientas tecnológicas puede ofrecer a los estudiantes un medio atractivo y motivador para fortalecer habilidades como las de indagación, y potenciar el aprendizaje de contenidos curriculares, además de maximizar los efectos en la mejora de las experiencias educativas del alumnado (Aguilera y Perales-Palacios, 2018; Busquets et al., 2016; Makokha, 2017). No obstante, las altas valoraciones que obtienen las actividades manipulativas y experienciales por parte de los estudiantes nos sugieren que ambos elementos, virtuales y manipulativos, interaccionan sinérgicamente y que aisladamente su 
efecto sería menor. Por tanto, tecnologías como la RVI no deberían sustituir a otras manipulativas con larga tradición en el ámbito de las ciencias, sino más bien complementarlas.

En lo referente a las limitaciones de este estudio, podemos mencionar el bajo número de participantes y la inexistencia de un verdadero grupo de control adecuado, con integrantes aleatorizados respecto al grupo experimental.

A modo general, concluimos que la integración de tecnologías de vanguardia, como es el caso de la RVI, constituye un elemento innovador y motivacional en la enseñanza de las disciplinas científico-matemáticas, especialmente porque posee la capacidad de presentar entornos simulados en los que el estudiantado tiene la posibilidad de interactuar y experimentar sin la necesidad de trasladarse fuera del centro educativo. No obstante, consideramos que su implementación debe ir acompañada de actividades que tengan la capacidad de articular lo experimentado dentro de la RVI con actividades escolares dirigidas a la enseñanza del currículo. En ese sentido, creemos que el enfoque STEM cumple con los requisitos para articular el uso de este tipo de tecnologías con metodologías de enseñanza activa.

A raíz de estas observaciones, se desprenden posibles líneas de investigación para el futuro:

a) Avanzar hacia una mayor integración de las diferentes disciplinas STEM, potenciando el pensamiento matemático y los procesos metacognitivos en relación con el mismo, al amparo de situaciones problemáticas que den sentido a lo que realiza el estudiante y creando tareas de indagación escolar.

b) Avanzar hacia una mayor integración de lo virtual con lo manipulativo y físico (que lo virtual nos lleve a realizar acciones y actividades en lo físico, y este, a su vez, en lo virtual).

\section{Referencias bibliográficas}

Aguilera, D. y Perales-Palacios, F.J. (2018). What Effects Do Didactic Interventions Have on Students' Attitudes Towards Science? A Meta-Analysis. Research in Science Education, 50, 573-597. <https://doi.org/10.1007/s11165-018-9702-2>

Akin, A. y Kurbanoglu, N. (2011). The relationships between math anxiety, math attitude and self-efficacy: A structural equation model. Studia Psychologica, 53(3), 263-273.

BAdeleH, A. (2019). The effects of robotics training on students' creativity and learning in physics. Education and Information Technologies. $<$ https://doi.org/10.1007/s10639-019-09972-6>

Busquets, T.; Silva, M. y Larrosa, P. (2016). Reflexiones sobre el aprendizaje de las ciencias naturales: Nuevas aproximaciones y desafíos. Estudios Pedagógicos, 42 (especial), 117-135. <https://doi.org/10.4067/s0718-07052016000300010>

BybeE, R. (2013). The case of STEM education: Challenges and Opportunities. Washington DC: National Science Teachers Association. 
Bybee, R. y McCrae, B. (2011). Scientific Literacy and Student Attitudes: Perspectives from PISA 2006 science. International Journal of Science Education, 33(1), 7-26. <https://doi.org/10.1080/09500693.2010.518644>

Caballero-García, P. y Fernández, T.G. (2019). Influence of maker-centred classroom on the students' motivation towards science learning. Cypriot Journal of Educational Science, 14(4), 535-544. <https://doi.org/10.18844/cjes.v11i4.4098>

Cabero, J. (coord.) (2015). Nuevas tecnologías aplicadas a la educación. Madrid: McGraw-Hill.

Cardona, M.C. (2002). Introducción a los métodos de investigación en educación. Madrid: EOS.

Chang, C.-C.; Tseng, K.-H. y Lou, S.-J. (2012). A comparative analysis of the consistency and difference among teacher-assessment, student self-assessment and peer-assessment in a Web-based portfolio assessment environment for high school students. Computers \& Education, 58(1), 303-320. <https://doi.org/10.1016/j.compedu.2011.08.005>

Chinn, S. (2012). Beliefs, Anxiety, and Avoiding Failure in Mathematics. Child Development Research, 1-8. $<$ https://doi.org/10.1155/2012/396071>

Cipresso, P.; Giglioli, I.A.C.; Raya, M.A. y Riva, G. (2018). The Past, Present, and Future of Virtual and Augmented Reality Research: A Network and Cluster Analysis of the Literature. Frontiers in Psychology, 9. <https://doi.org/10.3389/fpsyg.2018.02086>

Cohen, J. (1988). Statistical power analysis for the behavioral sciences. Nueva York: Lawrence Erlbaum Associates.

Confederación de Sociedades Científicas de España (COSCE) (2011). Informe Enciende. Madrid: COSCE.

Cremin, T.; Glauert, E.; Craft, A.; Compton, A. y Stylianidou, F. (2015). Creative Little Scientists: Exploring pedagogical synergies between inquiry-based and creative approaches in Early Years science. Education, 43(4), 404-419. <https://doi.org/10.1080/03004279.2015.1020655>

Escarbajal Frutos, A.; Essomba Gelabert, M.A. y Abenza Pastor, B. (2019). El rendimiento académico de alumnos de la ESO en un contexto vulnerable y multicultural. Educar, 55(1), 79-99. <https://doi.org/10.5565/rev/educar.967>

Fernández-Martín, F. D.; Arco-Tirado, J. L.; Hervás-Torres, M.; CarrilloRosúa, J.; Ruiz-Hidalgo, J. F. y Romero-López, M.C. (2020). Making STEM Education Objectives Sustainable through a Tutoring Program. Sustainability, 12, 6653. <https://doi.org/10.3390/su12166653>

Ferrada C.; Díaz-Levicoy, D.; Carrillo-Rosúa, J. y Silva-Díaz, F. (2020). La robótica desde las áreas STEM en Educación Primaria: una revisión sistemática. Education in the Knowledge Society (EKS), 21. <http://dx.doi.org/10.14201/eks.22036>

Flecha, R. y Puigvert, L. (2002). Las comunidades de aprendizaje: Una apuesta por la igualdad educativa. REXE: Revista de Estudios y Experiencias en Educación, 1(1), 11-20.

Freeman, A.; Adams, S.; Cummins, M.; Davis, A. y Hall, C. (2017). NMC/CoSN Horizon Report: 2017 K-12 Edition. Austin, Texas: The New Media Consortium. 
Gorard, S. y SEe, B.H. (2009). The impact of socio-economic status on participation and attainment in science. Studies in Science Education, 45(1), 93-129. <https://doi.org/10.1080/03057260802681821>

GweE, H.N. (2014). Effects of virtual-reality elements on spatial visualization skills of secondary three students in Singapore. En Y. CAI. 3D immersive and interactive learning (pp. 45-57). Singapur: Springer. <https://doi.org/10.1007/978-981-4021-90-6>

Hernández, R.; Fernández, C. y Baptista, P. (2014). Metodología de la investigación. 6. ${ }^{a}$ ed. México DF: McGraw-Hill.

Jiménez, M. (2008). Aproximación teórica de la exclusión social: Complejidad e imprecisión del término. Consecuencias para el ámbito educativo. Estudios Pedagógicos, 34(1), 173-186. <https://doi.org/10.4067/s0718-07052008000100010>

- (2012). Actuaciones socio-comunitarias y educativas inclusivas con alumnado en riesgo de exclusión social. Revista de Investigación en Educación, 10(2), 62-78.

León, O.G. y Montero, I. (2015). Métodos de investigación en Psicología y Educación: Las tradiciones cuantitativa y cualitativa. Madrid: McGraw-Hill.

Mакокна, J. (2017). Emerging Technologies and Science Teaching. En K.S. Taber y B. Akpan (eds.). Science Education (pp. 369-383). Róterdam: Sense Publishers.

Martín-Páez, T.; Aguilera, D.; Perales-Palacios, F.J. y Vílchez-González, J.M. (2019). What are we talking about when we talk about STEM education? A review of literature. Science Education, 103(4), 799-822. <https://doi.org/10.1002/sce.21522>

McDonAld, C. V. (2016). STEM Education: A review of the contribution of the disciplines of science, technology, engineering and mathematics. Science Education International, 27(4), 530-569.

Mata, M. de L.; Monteiro, V. y Peixoto, F. (2012). Attitudes towards Mathematics: Effects of Individual, Motivational, and Social Support Factors. Child Development Research, número especial, 1-10. <https://doi.org/10.1155/2012/876028>

Organización para la Cooperación y el Desarrollo Económico (2016). PISA 2015 Resultados Clave. Recuperado de <https://www.oecd.org/pisa/pisa-2015results-in-focus-ESP.pdf>.

Osborne, J.; Simon, S. y Collins, S. (2003). Attitudes towards science: A review of the literature and its implications. International Journal of Science Education, 25(9), 1049-1079. $<$ https://doi.org/10.1080/0950069032000032199>

Palacios, A.; Arias, V. y Arias, B. (2014). Las actitudes hacia las matemáticas: Construcción y validación de un instrumento para su medida. Revista de Psicodidáctica, 19(1), 67-91. <https://doi.org/10.1387/RevPsicodidact.8961>

Pozo, J.I. y Gómez, M.A. (1998). Aprender y enseñar ciencia. Madrid: Morata.

Radianti, J.; MajchrzaK, T.A.; Fromm, J. y Wohlgenannt, I. (2019). A systematic review of immersive virtual reality applications for higher education: Design elements, lessons learned, and research agenda. Computers \& Education, 103778. $<$ https://doi.org/10.1016/j.compedu.2019.103778>

Raposo-Rivas, M. y Martínez-Figueira, E. (2019). ¿Tecnologías emergentes o tecnologías emergiendo?: Un estudio contextualizado en la práctica preprofesional. Educar, 55(2), 499-518. <https://doi.org/10.5565/rev/educar.888> 
Rincón, D. del; Latorre, A.; Arnal, J. y Sans, A. (1995). Técnicas de investigación en ciencias sociales. Madrid: Dykinson.

Rocard, M. (2007). Science Education NOW: A renewed Pedagogy for the Future of Europe. Bruselas: European Commission.

Silva-Díaz, F; Fernández-Plaza, J. A. y Carrillo-Rosúa, J. (2019). Propuesta Didáctica. Realidad Virtual y STEM (REVI-STEM). Guía de implementación. Granada: Departamento de Didáctica de las Ciencias Experimentales - Universidad de Granada. Recuperado de <http://hdl.handle.net/10481/57160>.

SANDERs, M. (2009). STEM, STEM education, STEMmania. The Technology Teacher, 68(4), 20-27.

Schwarz, N. (2007). Attitude Construction: Evaluation in Context. Social Cognition, 25(5), 638-656. <https://doi.org/10.1521/soco.2007.25.5.638>

Sevian, H.; Dori, Y.J. y Parchmann, I. (2018). How does STEM context-based learning work: What we know and what we still do not know. International Journal of Science Education, 40(10), 1095-1107. <https://doi.org/10.1080/09500693.2018.1470346>

TARABini, A. (2017). La exclusión educativa: Significados, mecanismos y perfiles. En Los factores de exclusión educativa en España: Mecanismos, perfiles y espacios de intervención (pp. 6-25). Madrid: UNICEF.

Truchly, P.; Medvecky, M.; Podhradsky, P. y Vanco, M. (2018). Virtual Reality Applications in STEM Education. 2018 16th International Conference on Emerging eLearning Technologies and Applications (ICETA). <https://doi.org/10.1109/iceta.2018.8572133>

Vera, G.; Ortega, J.A. y Burgos, M.A. (2003). La realidad virtual y sus posibilidades didácticas.RevistaEtic@net,2(2).

Young, D. y Tamir, P. (1977). Finding out what Students know. The Science Teacher, 44, 27-28.

Zan, R. y Di Martino, P. (2007). Attitude toward Mathematics: Overcoming the positive/negative dichotomy. The Montana Mathematics Enthusiast (TMME), Monograph, 3, 157-168.

Zhang, D. y Campbell, T. (2011). The Psychometric Evaluation of a Three-Dimension Elementary Science Attitude Survey. Journal of Science Teacher Education, 22(7), 595-612.

<https://doi.org/10.1007/s10972-010-9202-3> 